\title{
Mapeamento de Patentes de Respiradores Artificiais ou Ventiladores Mecânicos
}

\author{
Patent Mapping of Artificial Respirators or Mechanical Fans
}

\author{
Edilson Araújo Pires ${ }^{1}$ \\ Jamile de Jesus Souza Andrade ${ }^{1}$ \\ Fábio André Lora ${ }^{1}$ \\ ${ }^{1}$ Universidade Federal do Recôncavo da Bahia, Feira de Santana, BA, Brasil
}

\begin{abstract}
Resumo
A pandemia da Covid-19 revelou uma carência de ventiladores mecânicos, equipamentos indispensáveis ao tratamento de pacientes que apresentam insuficiência respiratória, que tem sido a principal causa de morte daqueles infectados pelo Coronavírus. Esse artigo realizou um levantamento da produção de patentes relacionadas a ventiladores e respiradores utilizados no suporte ventilatório pulmonar. A coleta de dados foi realizada no Orbit Intelligence, empregando os termos (ventilat or respirat and mecanical) ou (artificial or medical), associados à Classificação Internacional de Patentes A61H-031/00; A61M-016/00. Foram analisadas 6.394 famílias de patentes, sendo o primeiro pedido requerido em 1909, mas, 2019 foi o ano mais produtivo. Foram identificadas 14 famílias de patentes de residentes brasileiros, evidenciando a necessidade de ampliar os estudos que garantam a evolução científico-tecnológica desses dispositivos no país. Por outro lado, existem mais de 5.900 famílias de patentes que podem ser exploradas no Brasil, sem infringir os direitos de propriedade industrial dos seus titulares.
\end{abstract}

Palavras-chave: Patentes. Respiradores Mecânicos. Coronavírus.

\begin{abstract}
The Covid-19 pandemic revealed a shortage of mechanical ventilators, essential equipment for the treatment of patients with respiratory failure, which has been the main cause of death for those infected with the Coronavirus. This article carried out a survey of the production of patents related to ventilators and respirators used in pulmonary ventilatory support. Data collection was carried out in Orbit Intelligence, using the terms (ventilat or respirat and mechanic) or (artificial or medical), associated with the International Patent Classification A61H-031/00; A61M016/00. 6,394 patent families were analyzed, the first being filed in 1909, but 2019 was the most productive year. 14 patent families of Brazilian residents were identified, highlighting the need to expand the studies that guarantee the scientific-technological evolution of these devices in the country. On the other hand, there are more than 5,900 patent families that can be exploited in Brazil, without infringing the industrial property rights of their owners.
\end{abstract}

Keywords: Patents. Mechanical Respirators. Coronavirus.

Área Tecnológica: Tecnologias Médicas. 


\section{Introdução}

As gripes com alta morbidade assustam a humanidade há bastante tempo. Em 1580, houve a primeira pandemia documentada, causada por um vírus do tipo influenza que surgiu na Ásia e se espalhou para a África, Europa e América do Norte (WHO, 2020a). De acordo com a World Health Organization (WHO, 2020a), a pandemia de 1918-1919, chamada de gripe espanhola, matou cerca de 40 milhões de pessoas em todo o mundo, e foi causada pelo vírus influenza A H1N1 que teve reincidência 90 anos depois, em 2009-2010, causando a nova pandemia, nomeada gripe suína.

Uma pandemia é a disseminação mundial de uma nova doença (WHO, 2020a). Nesse contexto, a pandemia é uma situação em que uma doença infecciosa ameaça, de forma simultânea, muitas pessoas ao redor do mundo.

Nos primeiros casos registrados da Covid-19 (Coronavirus Disease 2019), em dezembro de 2019, os pacientes apresentaram pneumonia viral em Wuhan, na China (XU et al., 2020). A disseminação da Covid-19 foi reconhecida como pandemia pela Organização Mundial de Saúde (OMS) no dia 11 de março de 2020 e tem assolado todo o mundo (SISTEMA UNIVERSIDADE ABERTA DO SUS, 2020). O Coronavírus já era conhecido pela ciência, embora provocasse apenas resfriados sem maiores complicações. Porém, com as mutações sofridas, surgiram sete cepas que são coronavírus humanos (HCoVs) e que acarretam danos graves à saúde: a) MERS-COV, que causa a síndrome respiratória do Oriente Médio; b) HCoV-OC43, HCoV-229E, HCoV-HKU1, HCoV-NL63, SARS-COV, que provocam síndrome respiratória aguda grave; e c) SARS-CoV-2, que causa a doença Covid-19 (PAHO, 2020).

Uma busca simples na base de dados de periódicos científicos Web of Science (CLARIVATE, 2021) com os termos "coronavirus and covid-19" demonstra a importância do tema para a comunidade científica ao redor do mundo. Em 16 de maio de 2021, já era possível resgatar 41.680 publicações, das quais, 29.729 e 11.949 foram publicadas em 2020 e 2021, respectivamente, e somente duas em 2019 devido à sua descoberta já no final do ano e às poucas informações existentes. Somente no Brasil, foram 1.577 publicações científicas. Essas evidências apontam, portanto, para o olhar dos pesquisadores na realização de pesquisas para compreender a Covid-19, suas implicações e possíveis estratégias e soluções para o combate à disseminação do vírus e para o tratamento da doença.

Em meados de 2020, a maioria dos países já se encontrava em um ponto de ruptura em termos de serviços de saúde (ARUNKUMAR et al., 2021). Em 16 de maio de 2021, havia 162.177.376 casos confirmados de Covid-19 no mundo notificados à Organização Mundial da Saúde, incluindo 3.364.178 mortes (WHO, 2021). O vírus atingiu todos os continentes do planeta e somente no Brasil, nessa mesma data, já havia 15.519 .525 casos confirmados e 432.628 mortes (WHO, 2021), concentrando, portanto, 9,56\% dos casos confirmados e $12,86 \%$ das mortes no mundo. Esses dados colocam o Brasil como o país em terceiro lugar no número de casos de Covid-19 confirmados e em segundo lugar no número de mortes causadas pela doença, o que torna preocupante a situação da pandemia no país (WHO, 2021).

A Covid-19 pode acarretar, em casos mais graves, febre, fadiga/mialgia e dispneia. Muitas vezes evoluindo para síndrome respiratória aguda grave (ISER et al., 2020). Assim, a disseminação rápida da doença ocasionou o aumento da demanda por ventiladores mecânicos $e$ por outros aparelhos e tecnologias que ajudam no tratamento de pacientes que apresentam 
insuficiência respiratória aguda ou crônica agudizada. $\mathrm{O}$ aumento da demanda revelou que o número de ventiladores mecânicos e de unidades de terapia intensiva nos sistemas de saúde é insuficiente para atender a um grande número de infectados ao mesmo tempo. No Brasil, por exemplo, em $72 \%$ das regiões, em 2020, o número de leitos de Unidades de Terapia Intensiva (UTIs) e de ventiladores e respiradores disponibilizadas pelo Sistema Único de Saúde (SUS) era inferior ao considerado adequado em um ano típico, sem a influência da Covid-19 (RACHE et al., 2020). De acordo com a Fundação Osvaldo Cruz (FIOCRUZ, 2021), em 3 de março de 2021, seguindo padrão preconizado pela OMS, a taxa de ocupação dos leitos de UTI apontava que apenas sete Unidades Federativas do país não havia atingido ainda a zona de alerta crítica (vermelho), quando as ocupações de UTI são iguais ou superiores a $80 \%$. Vale ressaltar, ainda, a inexistência de medicamento comprovadamente eficaz para o tratamento da Covid-19 e, embora haja o desenvolvimento de diversas vacinas que já estão sendo aplicadas na população, ainda vai demorar para vacinar todos.

O suporte ventilatório, conhecido como ventilação mecânica, consiste em um método de suporte para o tratamento e é aplicado com o objetivo de manter as trocas gasosas, aliviando o trabalho da musculatura respiratória; reverter ou impedir a fadiga da musculatura respiratória; e aumentar a oferta de oxigênio, dessa forma, reduzindo o desconforto respiratório (CARVALHO; TOUFEN JÚNIOR; FRANCA, 2007).

Desse modo, os ventiladores mecânicos ou respiradores artificiais aumentam as possibilidades de aplicação terapêuticas específicas sobre pacientes que possuem insuficiência respiratória durante o tratamento em Unidades de Terapia Intensiva (UTI), garantindo o fornecimento de ar e de oxigenação artificialmente (TOUFEN JÚNIOR; CARVALHO, 2007). Diante do contexto, a ventilação mecânica se tornou um dos principais métodos utilizados no tratamento de pacientes graves.

A ventilação mecânica ou artificial é realizada com a aplicação de pressão positiva nas vias aéreas e pode ser invasiva ou não invasiva (PLETSCH-ASSUNÇÃO; CUNHA; VIEIRA, 2019). Os autores destacam que a ventilação mecânica não invasiva, quando utilizada em grupos de pacientes bem selecionados, reduz a necessidade de entubação e a mortalidade. Já na ventilação mecânica invasiva, uma prótese (tubo oro ou nasotraqueal ou uma cânula de traqueostomia) é introduzida na via aérea, e, na ventilação não invasiva, uma máscara é empregada como interface entre o paciente e o ventilador mecânico (PLETSCH-ASSUNÇÃO; CUNHA; VIEIRA, 2019).

Entre os problemas relacionados à ventilação mecânica, destaca-se que, mesmo em circunstâncias ideais, a ventilação de um paciente com síndrome do desconforto respiratório agudo e doença pulmonar não homogênea está associada a uma taxa de mortalidade de 34\% a 60\% (RUBENFELD, 2003). Também não é recomendado o compartilhamento de aparelhos respiradores entre pacientes, visto que os equipamentos e as tecnologias atuais não garantem segurança nesse processo. Assim, nas decisões de triagem extremamente difíceis por escassez de aparelhos em número suficiente para atender a todos os pacientes, geralmente, as equipes médicas optam por dispor o ventilador ao paciente com maior probabilidade de recuperação.

Um mapeamento de patentes de ventiladores mecânicos foi realizado por Silva et al. (2020), no entanto, a pesquisa deles se limitou a realizar uma análise de 107 patentes concedidas e requeridas até o ano de 2000. Dessa forma, uma lacuna deixada pelo trabalho se refere a uma análise mais aprofundada do estado da técnica, não somente para identificação de tecnologias já protegidas e que impedem a apropriação de inventos que infringem essas tecnologias (por 
exemplo, pedidos de patentes publicados), mas também um levantamento daquelas tecnologias que não foram requeridas patentes no Brasil e, portanto, podem ser apropriadas e colocadas no mercado brasileiro sem infringir direitos de propriedade intelectual.

Analisando o avanço da atual pandemia e a importância dos respiradores mecânicos no enfrentamento à Covid-19, um estudo que faça o levantamento das tecnologias (patentes) de ventilação mecânica pulmonar no mundo é de grande relevância. Nesse contexto, os questionamentos apresentados por Suzuki e Quintella (2020, p. 311), em destaque "b) Será que não seria melhor aprender, por exemplo, com patentes já publicadas, com seus inventores ou titulares?", são balizadores desta pesquisa, pois se trata de construção de conhecimento por meio de pesquisas associadas à busca de anterioridade e prospecção tecnológica de patentes. Suzuki e Quintella (2020, p. 311) afirmam que as iniciativas no Brasil para desenvolvimento de tecnologias para auxiliar no tratamento da doença, como os ventiladores pulmonares, frente à desindustrialização e à perda de capacidade produtiva do país, têm evidenciado o grau de despreparo tecnológico da nação brasileira.

Os resultados deste estudo podem, portanto, contribuir para a tomada de decisão dos pesquisadores e de empresas que atuam no desenvolvimento de tecnologias médicas e, também, para os agentes e organizações de saúde na adoção de tecnologias já existentes.

Este trabalho realiza um mapeamento da produção de patentes relacionadas a ventiladores e respiradores utilizados no suporte ventilatório pulmonar, a nível mundial. Justifica-se, por apresentar à sociedade dados relacionados à tecnologia dos respiradores, especialmente, neste momento em que a pandemia de Covid-19 aumentou muito a demanda por esses aparelhos. Ademais, a pesquisa servirá de suporte para futuros projetos.

De acordo com o Instituto Nacional da Propriedade Industrial (INPI, 2020), patente é um título de propriedade temporária sobre uma invenção ou modelo de utilidade, outorgado pelo Estado aos inventores ou autores ou a outras pessoas físicas ou jurídicas detentoras de direitos sobre a criação. $\mathrm{O}$ inventor ou o detentor da patente tem direitos sobre o objeto de sua patente e/ou processo ou produto obtido diretamente por processo por ele patenteado. Em contrapartida, o inventor se obriga a revelar detalhadamente todo o conteúdo técnico da matéria protegida pela patente (INPI, 2020). Assim, as patentes são importantes fontes de informação sobre os avanços tecnológicos nas diversas áreas.

A recuperação de patentes é um subdomínio da recuperação de informações, cujos elementos básicos de pesquisa são documentos de patentes que, devido às características desses documentos e aos requisitos especiais de recuperação, as pesquisas nas inúmeras bases de referência de documentos de patentes são, geralmente, mais longas e complexas do que os das pesquisas na web (ZHANG; LI; LI, 2015).

A pesquisa de patente, na prática, tem cinco tarefas representativas:

a) Pesquisa de arte prévia, que visa a compreender o estado da arte de um tópico geral ou uma tecnologia direcionada, assim o escopo dessa tarefa busca todas as publicações disponíveis em todo o mundo.

b) Pesquisa de patenteabilidade, que tenta recuperar os dados relevantes de documentos em todo o mundo que já foram publicados até a data de aplicação, e pode divulgar o conceito central da invenção. 
c) Pesquisa de invalidez, que pesquisa as publicações disponíveis que invalidam um documento de patente publicado. Essa tarefa geralmente é realizada após a concessão de uma patente.

d) Pesquisa de violação, que recupera publicações de patentes válidas que são infringidas por um determinado produto ou documento de patente. Em geral, a pesquisa opera na reivindicação da seção dos documentos de patentes disponíveis.

e) Pesquisa de status legal, que determina se uma invenção tem liberdade para fazer, usar e vender; isto é, se a patente concedida expirou ou não (ZHANG; LI; LI, 2015).

Adicionalmente, conforme apontam Pires, Ribeiro e Quintella (2020, p. 14), as informações contidas em um documento permitem também uma análise de

[...] dados dos recursos humanos envolvidos na criação da tecnologia, das organizações titulares daquela tecnologia, datas em que as patentes foram requeridas, os países que são foco da proteção (potenciais mercados), a anterioridade do estado da arte de cada invenção, entre outros.

\section{Metodologia}

Este estudo é caracterizado como descritivo exploratório e de abordagem quantitativa. Nesse caso, para realizar as tarefas de pesquisa de patentes, a metodologia seguiu o procedimento de pesquisa de patentes ilustrado na Figura 1.

Figura 1 - Procedimento da pesquisa de patentes

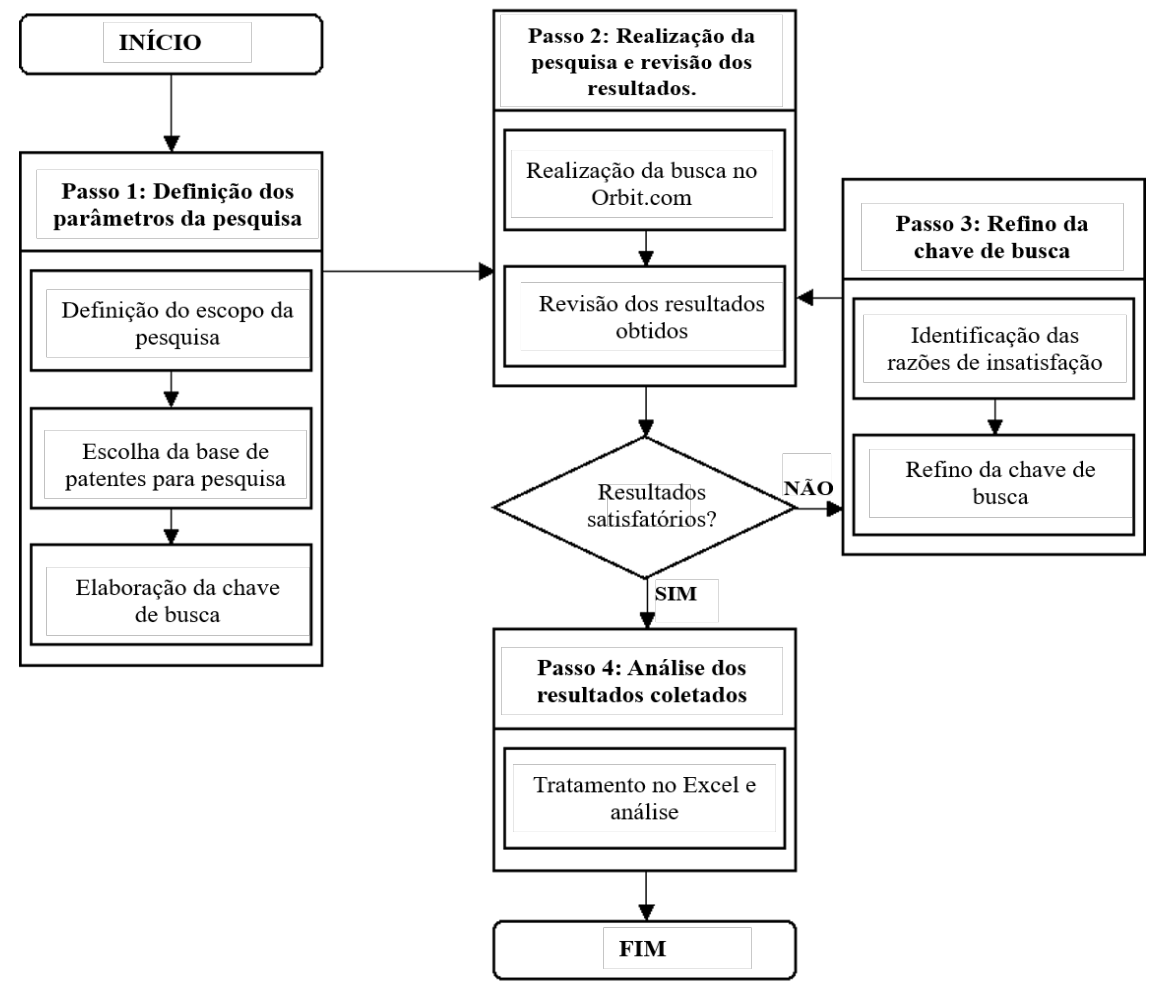

Fonte: Adaptada de Zhang, Li e Li (2015) 
No primeiro passo da pesquisa, foram definidos os parâmetros para a consulta de recuperação de patentes, ou seja, a definição da chave de busca inicial. A pesquisa buscou recuperar documentos relevantes publicados antes da data da pesquisa, assim o escopo de pesquisa abrange todos os documentos disponíveis em todo o mundo. Após a realização das primeiras consultas e revisões dos resultados, foi necessário refinar a chave de busca a fim de melhorar os resultados capturados. Assim, após o refinamento da chave de busca, foi possível alcançar resultados satisfatórios para a análise de acordo com o objetivo proposto.

Nesse contexto, buscou-se apresentar o mapeamento da produção de patentes relacionadas a respiradores e ventiladores mecânicos. Para tanto, a metodologia da pesquisa foi realizada por meio da busca no estado da técnica, mediante consulta no Orbit.com - Questel Orbit Intelligence ${ }^{\circledR}$ - que é uma base on-line de patentes da Questel Orbit, Inc, empresa franco-americana destacada como uma das líderes globais nesse segmento desde a década de 1970. O Orbit. com permite buscar, selecionar, analisar e exportar informações contidas em patentes e foi escolhido por ter uma abrangência mundial. Em fevereiro de 2018, o sistema Orbit contava com 58,185 milhões de famílias de patentes e mais de 107 milhões de documentos individuais de patentes, fornecidas por 107 jurisdições de patentes (PIRES; RIBEIRO; QUINTELLA, 2020). Desse modo, se as ferramentas de buscas forem outras, podem ocorrer resultados diferentes dos encontrados neste trabalho.

O levantamento de patentes utilizado para este trabalho foi feito utilizando-se as seguintes palavras-chave e operadores booleanos: "(ventilat or respirat) and (mecanical or artificial or medical)", associadas aos termos "(system and control)", restringindo-se a busca aos documentos que apresentaram os termos no título e/ou resumo e, de forma complementar aplicou-se também o filtro de Classificação Internacional de Patentes (CIP) A61H-031/00 (Respiração artificial ou estimulador do coração) or A61M-016/00 (Dispositivos para influenciar o sistema respiratório de pacientes por tratamento com gás, e respiração boca a boca; Tubos traqueais (estimulando os movimentos respiratórios por meios mecânicos, pneumáticos ou elétricos, pulmões de ferro combinados com meios de respiração de gás) para restringir a pesquisa aos respiradores e ventiladores mecânicos. A Classificação Internacional de Patentes (CIP), constituída pelo Acordo de Estrasburgo de 1971, é um sistema hierárquico de símbolos para a classificação de patentes e de modelos de utilidade de acordo com as diferentes áreas de tecnologia a que pertencem (INPI, 2020).

Cerca de 6.397 patentes atendiam aos termos da pesquisa. O levantamento foi realizado no mês de setembro de 2020, mas a revisão dos resultados foi realizada na primeira semana de março de 2021. Para fins desta pesquisa, consideram-se como unidade de referência as famílias de patentes, que são reconhecidas nesta pesquisa como um conjunto de documentos de patentes que partilham a mesma prioridade e que podem se referir a pedidos de patente em processo de exame ou patentes concedidas, expiradas, revogadas, ou seja, independentemente do seu estado legal (ativas ou inativas).

As patentes foram tratadas pela inserção de seus dados em gráficos plotados em planilha eletrônica para a devida análise. Na análise, foi dada maior ênfase para os dados referentes aos últimos 50 anos (1970-2019), período que apresenta maior frequência no depósito de patentes relacionadas à tecnologia pesquisada. 


\section{Resultados e Discussão}

Para a análise da evolução anual de depósito de famílias de pedidos de patentes, o levantamento considerou o período desde o primeiro depósito, em 1909, até o ano de 2019. O primeiro registro de pedido de patente que atende aos critérios da busca foi depositado em 22 de janeiro de 1909 e publicado no ano seguinte, tendo como objeto, conforme documento de patente GB190901638, inspirador e respirador artificiais combinados para restaurar a animação em pessoas ou animais aparentemente afogados ou asfixiados, de titularidade e inventado por Gray Oliver e com proteção requerida apenas na Gram Betânia. No entanto, cabe destacar que, segundo Kacmarek (2011), já eram conhecidos na segunda metade do século XVIII aparelhos mecânicos para fornecer ventilação não invasiva de pressa positiva.

Cabe destacar um fato importante ocorrido em meados do século XX e que revolucionou a tecnologia médica no desenvolvimento de respiradores e ventiladores artificiais mecânicos: a criação do Ambu (Artificial Manual Breathing Unit). Conforme destacado por Spricido (2021), o Ambu nada mais é do que uma Unidade Manual de Respiração Artificial, ou Reanimador Manual. Esse equipamento é composto de um balão, uma válvula para reservatório (válvula unidirecional que impede que o ar exalado pelo paciente retorne ao balão), uma máscara facial e um reservatório. É utilizado para fornecer ventilação com pressão positiva a pacientes com necessidade de suporte ventilatório, sendo o principal dispositivo para ventilação durante a reanimação cardiopulmonar. Também fornece oxigênio aos pacientes, substitui temporariamente a ventilação mecânica, ventila pacientes durante o transporte intra e extra-hospitalar, e é utilizado na fisioterapia respiratória. Equipamentos de ventilação artificial portáteis só passaram a ser utilizados rotineiramente em 1952, durante epidemia de poliomielite. Em 1956, a empresa Ambu International inventou o primeiro reanimador portátil autoinflável, com válvula de não reinalação, que recebeu o nome de Ambu (SPRICIDO, 2021).

Para melhor entendimento da evolução e do caminho do desenvolvimento tecnológico percorrido por tecnologias associadas ao nosso escopo de pesquisa, foi dada maior ênfase à análise das famílias de pedidos de patentes depositadas nos últimos 50 anos e já publicadas na data de realização da pesquisa (1970-2019) - 6.025 famílias de pedidos de patentes, cerca de $94 \%$ do total de dados da pesquisa - pois, nesse período, nota-se o crescente interesse pela proteção de invenções relacionadas a respiradores e ventiladores mecânicos, conforme ilustra Figura 2. Vale ressaltar que, no ano de 2019, podem existir pedidos ainda não publicados, isso ocorre devido ao período de sigilo de 18 meses. 
Figura 2 - Evolução anual das famílias de patentes de respiradores artificiais e ventiladores mecânicos com primeira prioridade requerida entre 1970 e 2019

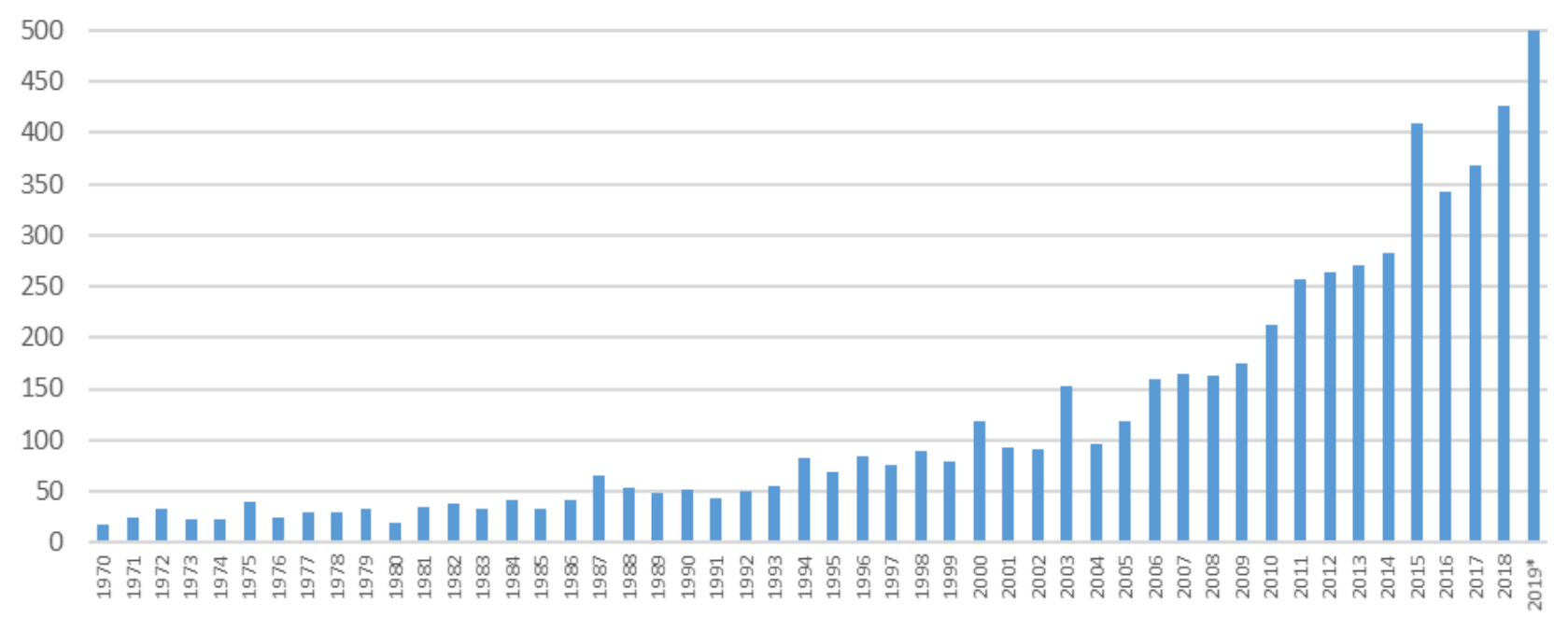

*Devido ao período de sigilo estabelecido de 18 meses contados a partir da data de propriedade do pedido de patente, o número apresentado em 2019 pode ser maior do que o encontrado nesta figura.

Fonte: Elaborada pelos autores deste artigo (2021)

Observa-se na Figura 2 que, nos últimos 10 anos analisados, o número de famílias de patentes requeridas anualmente quase triplicou, período que corresponde a $55 \%$ de todas as famílias de pedidos de patentes requeridas entre 1970 e 2019. Nota-se, também, que, a partir de 2008, parece haver uma tendência de crescimento mais acentuada, com o maior número de pedidos de patentes em 2019, exceto para o ano de 2016, pois, houve declínio no número de famílias de pedidos de patentes requeridas em relação ao ano anterior.

Quando se compara o número de famílias de patentes com o número de publicações científicas publicadas ao redor do mundo e que tratam de ventiladores mecânicos, são identificadas, entre 1945 e 16 de maio de 2021, 2.382 publicações, segundo a base de dados Web of Science (CLARIVATE, 2021). Cabe destacar que o ano de 2020 foi quando mais artigos científicos foram publicados sobre o tema pesquisado, 131, e, ainda, 16 publicações têm alguma citação do termo Covid-19.

No que se refere aos residentes brasileiros, foram identificadas 14 famílias de pedidos de patentes protocoladas no período em análise. $\mathrm{O}$ primeiro pedido de Prioridade $\mathrm{BR}$ foi requerido somente em 1993 e se tratava de "Um sistema de controle de ciclo respiratório", de titularidade da Intermed Equipamento Médico Hospitalar Ltda (PI9304638-3B1). Essa patente foi concedida no Brasil em 17/12/1999 e extinta em 6/12/2013, de modo que desde essa última data passou a ser de domínio público, podendo ser explorada por qualquer empresa. Nessa invenção, torna-se relevante destacar o inventor da tecnologia, Jorge Bonassa, pois, conforme descrito no seu currículo lattes (http://lattes.cnpq.br/7373617216548749), trata-se de um Engenheiro Mecânico, com doutorado em Ciências Médicas e Biológicas, que dedicou sua vida profissional, trabalhando para Intermed por 30 anos (1985-2015), ao desenvolvimento de diversas tecnologias no campo da ventilação mecânica pulmonar e anestesia, portanto, ligadas diretamente ao 
objeto de estudo desta pesquisa. Trata-se aqui de um destaque preponderante, especialmente porque o número de doutores empregados em empresas brasileiras é relativamente pequeno. Conforme destacam Galvão et al. (2016), em 2014, 87\% dos doutores eram empregados nas atividades de Educação e Administração Pública e apenas 9\% eram empregados em atividades profissionais, científicas e técnicas e indústrias de transformação. Esses dados apontam que, apesar de Silva et al. (2020) destacarem que não foram identificados documentos e patentes relacionadas a ventiladores mecânicos de titularidade de residentes no Brasil, o desenvolvimento dessa tecnologia já fazia parte do escopo de Pesquisa e Desenvolvimento (P\&D) de organizações localizadas no país.

Já o último pedido de patente (BR102019000814) de um residente brasileiro e com prioridade BR foi requerida em 15/01/2019, pela Universidade Federal de Pernambuco (UFPE). O documento refere-se a "Dispositivo e método de medição e controle da pressão de balonete de próteses traqueais" e ainda está em julgamento pelo INPI do Brasil, mas pode ser considerado uma importante tecnologia para auxiliar no tratamento de pacientes infectados pela Covid-19.

$\mathrm{O}$ ano de 2007 foi o que teve mais pedidos de patentes protocolados com prioridade $\mathrm{BR}$, foram quatro processos.

A corrida pelo desenvolvimento de equipamentos médicos, como os ventiladores mecânicos, tem estimulado a adoção de medidas para dar mais celeridade à regulação desses dispositivos pela Agência Nacional de Vigilância Sanitária (ANVISA). Uma dessas medidas foi a publicação da Resolução da Diretoria Colegiada (RDC) n. 356/2020, que flexibiliza, simplifica e agiliza processos de regularização sanitária de equipamentos médicos (ANVISA, 2020). Outra medida adotada pelo órgão foi a maior dedicação da Gerência Geral de Inspeção e Fiscalização Sanitária (GGFIS) e da Gerência Geral de Produtos para Saúde (GGTPS) para dar celeridade na avaliação dos projetos prioritários e ampliar a capacidade produtiva dos ventiladores pulmonares no Brasil, tendo em vista que esse é um dos aparelhos mais mencionados quando o assunto é o novo coronavírus (ANVISA, 2020).

Segundo Santos (2020), instituições de pesquisa e universidades brasileiras estão apresentando iniciativas de desenvolvimento de novos respiradores, como a Universidade Federal da Paraíba, Serviço Nacional de Aprendizagem Industrial (SENAI) do Amazonas, Escola Politécnica (Poli) da Universidade de São Paulo, o Instituto Alberto Luiz Coimbra de Pós-Graduação e Pesquisa de Engenharia, da Universidade Federal do Rio de Janeiro, e a Petrobrás. Essas informações apontam para um cenário futuro em que o número de patentes brasileiras desses dispositivos seja mais significativo, e a dependência por tecnologia internacional diminua.

A Figura 3 apresenta o status legal das famílias de patentes de ventiladores mecânicos depositadas no mundo entre 1970 e 2019. 
Figura 3 - Status legal das famílias de patentes de respiradores artificiais e ventiladores mecânicos com primeira prioridade requerida entre 1970 e 2019

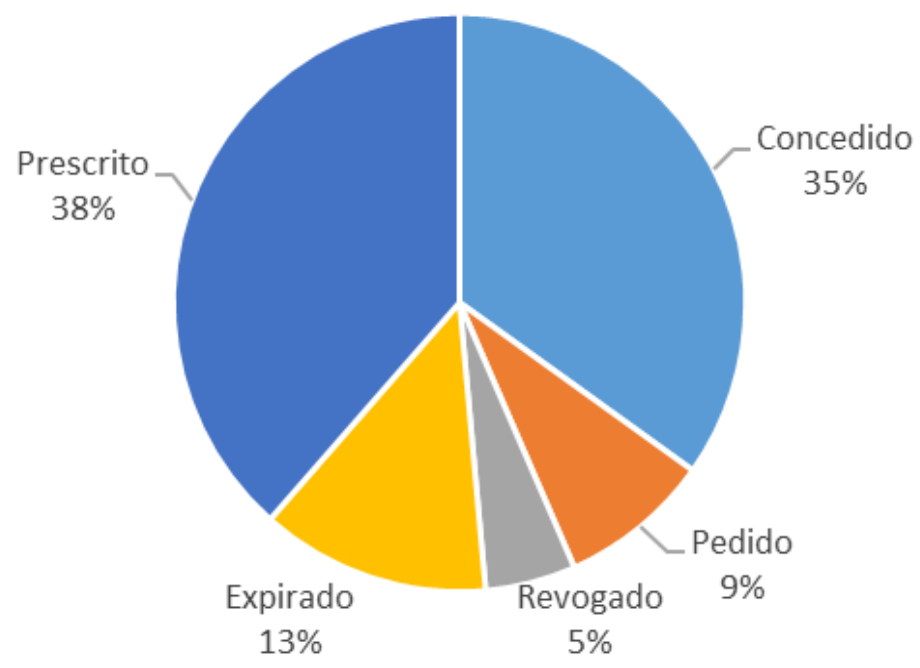

Fonte: Elaborada pelos autores deste artigo (2021)

Esses dados apresentados na Figura 3 trazem embutidos uma importante informação acerca da possibilidade de exploração das patentes prescritas, expiradas e revogadas, cerca de $57 \%$ do total pesquisado ou 3.408 tecnologias, sem que haja necessidade de requerer autorização para explorar essas tecnologias no mercado ou pagar royalties pela sua exploração. Das 6.025 famílias de patentes, 2.617 são famílias de patentes que têm seus direitos de propriedade industrial preservados, pois, ou estão em análise ou já foram concedidas as cartas patentes e estão dentro do prazo de vigência. No entanto, como as patentes são direitos territoriais, no que se refere ao Brasil, apenas 114 invenções têm seus direitos de propriedade industrial vigentes no país, de modo que mais de 5.900 invenções relativas a respiradores e ventiladores podem ser exploradas no mercado brasileiro.

Como a exploração dos respiradores e ventiladores depende de regulação da Anvisa, por se tratar de equipamentos de alto risco e de suporte à vida dos pacientes, esses equipamentos precisam passar por um registro junto à Agência. Para tanto, devem ser cumpridos os requisitos previstos na legislação vigente, especificadas nas RDCs n. 56/2001 e n. 185/2001, além dos requisitos apresentados na Norma Técnica NBR ISO 80601-2-12 e suas normas complementares de segurança elétrica e compatibilidade eletromagnéticas, bem como a comprovação de evidência clínica de segurança e desempenho mínimo em pacientes (pesquisa clínica em pacientes, conforme RDC n. 10/2015 e RDC n. 375/2020) (ANVISA, 2020). Com a finalidade de dar celeridade a esse processo, a Anvisa publicou a RDC n. 349/2020, que simplifica os requisitos para registro de ventiladores pulmonares já registrados e comercializados nos países membros do International Medical Device Regulators Forum, ou seja, Estados Unidos da América, Canadá, Comunidade Europeia, Austrália, Singapura, Japão, China, Coreia do Sul, Rússia e Brasil (ANVISA, 2020).

As 6.025 famílias de patentes analisadas têm participação como titulares 1.249 organizações, das quais, 11 são organizações sediadas no Brasil. Ainda acerca dos titulares, 121 são universidades e, destas, três são brasileiras. Os principais titulares das famílias de patentes, com posição estratégica de proteção da propriedade industrial, são apresentados na Tabela 1. A maioria das organizações atua no setor de tecnologias médicas. 
Tabela 1 - Principais titulares das famílias de patentes de respiradores artificiais e ventiladores mecânicos com primeira prioridade requerida entre 1970 e 2019

\begin{tabular}{cccc} 
EmpRESAS & $\begin{array}{c}\text { FAMíliAS DE } \\
\text { PATENTES }\end{array}$ & EMPRESAS & $\begin{array}{c}\text { FAMíliAS DE } \\
\text { PATENTES }\end{array}$ \\
Philips & 204 & Excelsior Union & 21 \\
Fps Vermögensverwaltung & 148 & Nanjing Medical University & 21 \\
Medtronic & 93 & Weinmann Gerate Fur Medizin & 21 \\
Resmed & 93 & Hunan Zhenghong Science \& & 20 \\
Asahi Kasei & 61 & Technology Development & 19 \\
Air Liquide & 56 & Mallinckrodt & 19 \\
General Electric & 55 & Siemens & 19 \\
Fisher \& Paykel Healthcare & 50 & Suzuki Motor & 18 \\
Universal Robot Systems & 46 & Jilin University & 17 \\
Becton Dickinson \& Company & 42 & Bank of New York Mellon & 17 \\
Teijin & 27 & Predpriyatie P Ya A 1001 & 17 \\
Aeonmed & 26 & Teleflex & 17 \\
Loewenstein Helmut & 24 & Yinzhou People S Hospital & 16 \\
Avanos Medical & 22 & Air Water & 16 \\
Beijing Aerospace Changfeng & 22 & Linde & 15 \\
\hline \hline
\end{tabular}

Fonte: Elaborada pelos autores deste artigo (2021)

As 30 organizações listadas na Tabela 1, apesar de representarem apenas 2,4\% dos titulares, detêm, juntas, $20,5 \%$ de todas as famílias de patentes relacionadas a respiradores e ventiladores mecânicos depositadas no mundo entre 1970 e 2019. A Philips se destaca entre os titulares, pois detém $6,75 \%$ de todas as patentes relacionadas a essas tecnologias nos últimos 50 anos. A Philips (ou Royal Philips) é uma empresa sediada na Holanda e atua no desenvolvimento de tecnologias diversificadas, especialmente nas áreas de Cuidados com a saúde, Estilo de vida e Iluminação. Somente em 2019, a empresa investiu 1,9 bilhão de euros em Pesquisa e Desenvolvimento (P\&D) e foi classificada pelo sétimo ano consecutivo entre as empresas mais inovadoras do mundo pelo Top 100 Global Innovators Clarivate (https://clarivate.com/top-100-innovators/ the-top-100/philips/). Seguido da Philips, aparece a FPS Vermögensverwaltung, com 148 (4,9\%), e a Medtronic e Resmed, com 93 (3,1\%). Ainda se destacam, entre as organizações citadas na Tabela 1, a Nanjing Medical University e Jilin University, ambas chinesas, por estarem entre as 25 maiores detentoras de direitos de propriedade industrial com famílias de patentes relacionadas a respiradores e ventiladores mecânicos requeridas entre 1970 e 2019.

No Brasil, a Philips também é a maior detentora dos direitos de propriedade industrial de patentes referentes a ventiladores e respiradores artificiais, figurando como titular de 41 das 114 patentes ativas no país. Em seguida, estão a Altria Group e a Air Liquid, ambas como titulares de sete patentes. Na lista de tutelares de patentes de respiradores artificiais ou ventiladores mecânicos, ativas no Brasil, têm destaque a Universidade Federal do Ceará (UFC) e a Universidade Federal de Pernambuco (UFPE), ambas com apenas um pedido de patente. 
Na Tabela 2, são apresentados os principais inventores das famílias de patentes de respiradores artificiais ou ventiladores mecânicos com primeira prioridade requerida entre 1970 e 2019.

Tabela 2 - Principais inventores das famílias de patentes de respiradores artificiais e ventiladores mecânicos com primeira prioridade requerida entre 1970 e 2019

\begin{tabular}{|c|c|c|}
\hline INVENTORES & Famílias de Patentes & ORganizaÇÃo dE TITULARIDADE DAS PATENTES \\
\hline Freeman Gary A & 25 & Asahi Kasei \\
\hline Dai Zheng & 20 & Hunan Zhenghong Science \& Technology Development \\
\hline Diao Jun & 20 & Beijing Aerospace Changfeng \\
\hline Truschel William Anthony & 18 & Philips \\
\hline Zhuang Zhi & 18 & BMC Médical Co \\
\hline Sugiura Yasuhito & 17 & Suzuki Motor; Metran \\
\hline Jafari Mehdi M. & 15 & Medtronic \\
\hline Schwaibold Matthias & 14 & Loewenstein Helmut; Weinmann Gerate Fur Medizin \\
\hline Jehmlich Klaus & 13 & Bank Of New York Mellon \\
\hline Richard Jean-Christophe & 13 & Air Liquide \\
\hline Tham Robert Q. & 13 & General Electric \\
\hline Gobel Christof & 13 & Loewenstein Helmut; Weinmann Gerate Fur Medizin \\
\hline Heesch Ralf & 13 & Fps Vermögensverwaltung \\
\hline Nitsuta Kazutoshi & 13 & Applied Materials; Suzuki Motor; Metran \\
\hline Wedler Wolfgang & 12 & Loewenstein Helmut; Weinmann Gerate Fur Medizin \\
\hline Zhang Wei & 12 & Zunyi Medical university \\
\hline Li Yan & 12 & Philips \\
\hline Ahmad Samir & 11 & Philips \\
\hline Huby Ronald James & 11 & Resmed \\
\hline Hansmann Hans-Ullrich & 11 & Fps Vermögensverwaltung \\
\hline Feng Gengchao & 11 & Boomsense Technology \\
\hline Kim Jong Cheol & 11 & MEK \\
\hline Liu Wei & 11 & Hunan Zhenghong Science \& Technology Development \\
\hline Yasukawa Mikio & 11 & Suzuki Motor; Metran \\
\hline Bath Andrew Roderick & 10 & Resmed \\
\hline Nitta Kazufuku & 10 & Metran \\
\hline Acker Jaron M. & 10 & General Electric \\
\hline Ahmed Sami & 10 & Philips \\
\hline
\end{tabular}

Fonte: Elaborada pelos autores deste artigo (2021)

Como era de se esperar, os principais inventores são em sua maioria ligados a empresas que fabricam dispositivos médicos e soluções de software, como é o caso do Freeman Gary, que figura como inventor de $40 \%$ de todas as famílias de patentes de titularidade da empresa Zoll 
Medical Corporation, adquirida em 2012 pelo Grupo Asahi Kasei, que tem sede no Japão e atua em diversos seguimentos da Economia. Empatados com a mesma quantidade de patentes depositadas estão Dai Zheng, da Hunan Zhenghong Science \& Technology Development, e Diao Jun, da Beijing Aerospace Changfeng Co, ambas organizações chinesas. Como se verifica na Tabela 2, a Hunan Zhenghong Science \& Technology Development aparece como titular de 20 famílias de patentes, Dai Zheng, portanto, atuou no desenvolvimento de todas as tecnologias de ventiladores e respiradores patenteadas pela empresa em questão. Em seguida, aparecem Truschel William Anthony, ligado a Philips, e Zhuang Zhi, da BMC Medical Co. Conforme apresentado em seu site, a BMC Medical foi fundada em 2001 em Pequim para fornecer soluções integradas para distúrbios respiratórios do sono (DRS) e outras doenças respiratórias crônicas, portanto, especializada no desenvolvimento das tecnologias aqui prospectadas.

Entre os inventores que figuram nas 14 famílias de patentes de prioridade brasileira, Jorge Bonassa se destaca com cinco patentes, estas com os direitos de propriedade industrial pertencentes a Becton Dickinson \& Company, empresa que incorporou a Intermed Equipamento Médico Hospitalar. A Becton é uma empresa multinacional americana especializada na fabricação de dispositivos médicos, sediada nos Estados Unidos da América.

A informação tecnológica referente ao país de prioridade no documento de patente permite analisar quais os países com desenvolvimento tecnológico mais significativo em um determinado setor. Considerando-se as 6.021 famílias de patentes abrangidas neste estudo, a distribuição dos escritórios de propriedade industrial e territórios de prioridade está representada na Figura 4.

Figura 4 - Famílias de patentes de respiradores artificiais e ventiladores mecânicos com primeira prioridade requerida entre 1970 e 2019, por primeiro país de prioridade
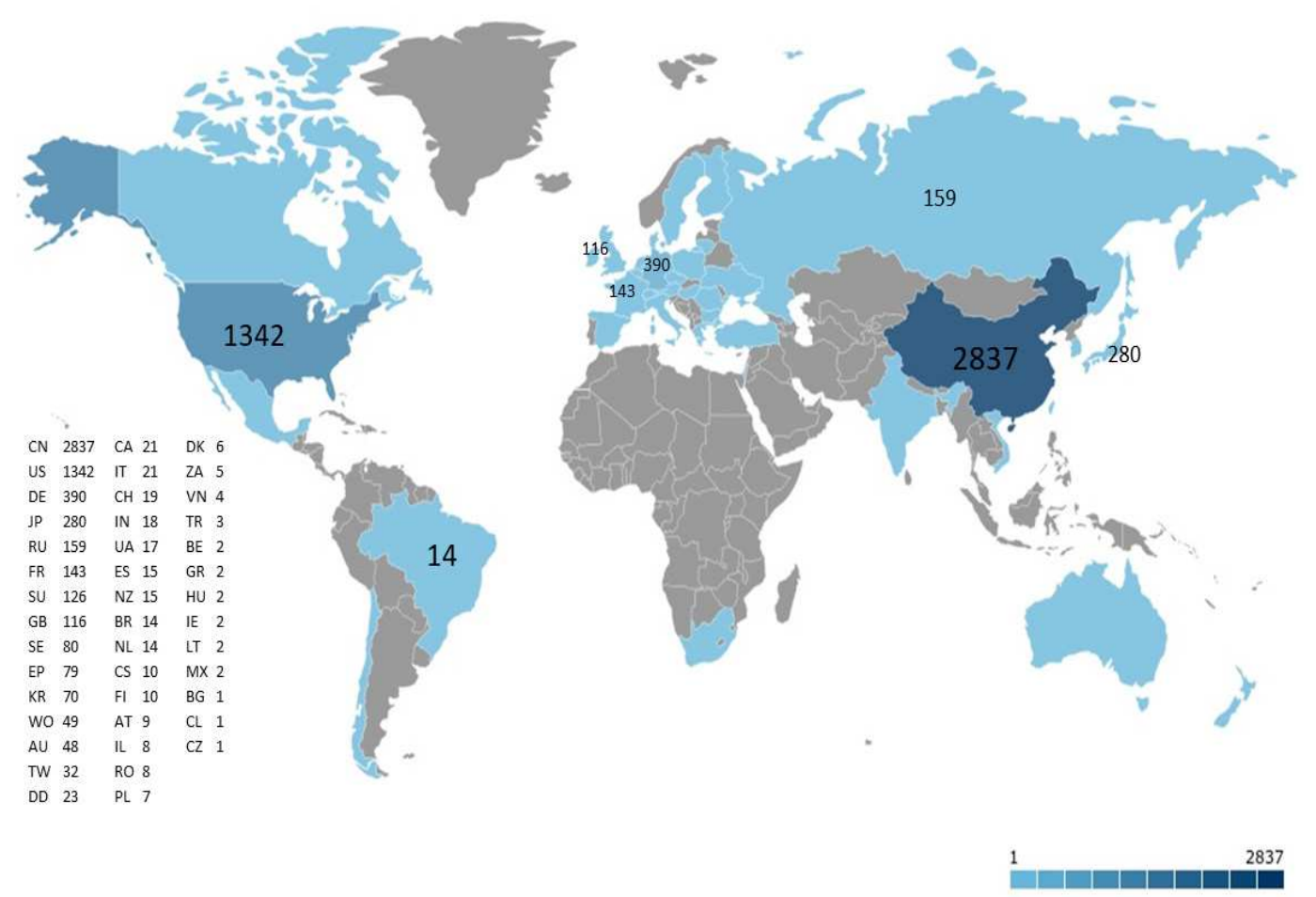

Fonte: Elaborada pelos autores deste artigo (2021)

A China $(\mathrm{CN})$ detém $47 \%$ das famílias de patentes requeridas no mundo acerca de ventiladores ou respiradores mecânicos, seguida pelos Estados Unidos, com 22\%. Já o Brasil (BR) possui 
apenas $0,23 \%$ das famílias de pedidos de patentes requeridas com prioridade $\mathrm{BR}$, colocando o país apenas em $21^{\circ}$ no ranking de países requerentes de patentes relacionadas a ventiladores/ respiradores mecânicos, o que evidencia uma deficiência do país em pesquisa e desenvolvimento tecnológico nessa área e o coloca refém da aquisição de tecnologias desenvolvidos por organizações de outros países.

Por outro lado, ao considerar as famílias de patentes ativas, ou seja, aquelas que estão em análise (pedidos de patentes) e as concedidas que estão vigentes (ainda não completaram 20 anos da data de prioridade e já foram deferidas), observa-se na Figura 5 que, apesar de o Brasil possuir apenas 14 famílias de patentes de prioridade BR, existem 114 famílias de patentes com seus direitos vigentes. Essas informações colocam o País na $17^{a}$ colocação entre os mercados de exploração da tecnologia aqui prospectada, com apenas $1,4 \%$ do total de família de patentes ativas no mundo. $\mathrm{O}$ número reduzido de proteção no Brasil indica que, em escala mundial, o país não é considerado um mercado promissor pelos inventores e depositantes.

Figura 5 - Famílias de patentes ativas (pedidos e concedidas) de respiradores artificiais e ventiladores mecânicos com primeira prioridade requerida entre 1970 e 2019, por país de proteção

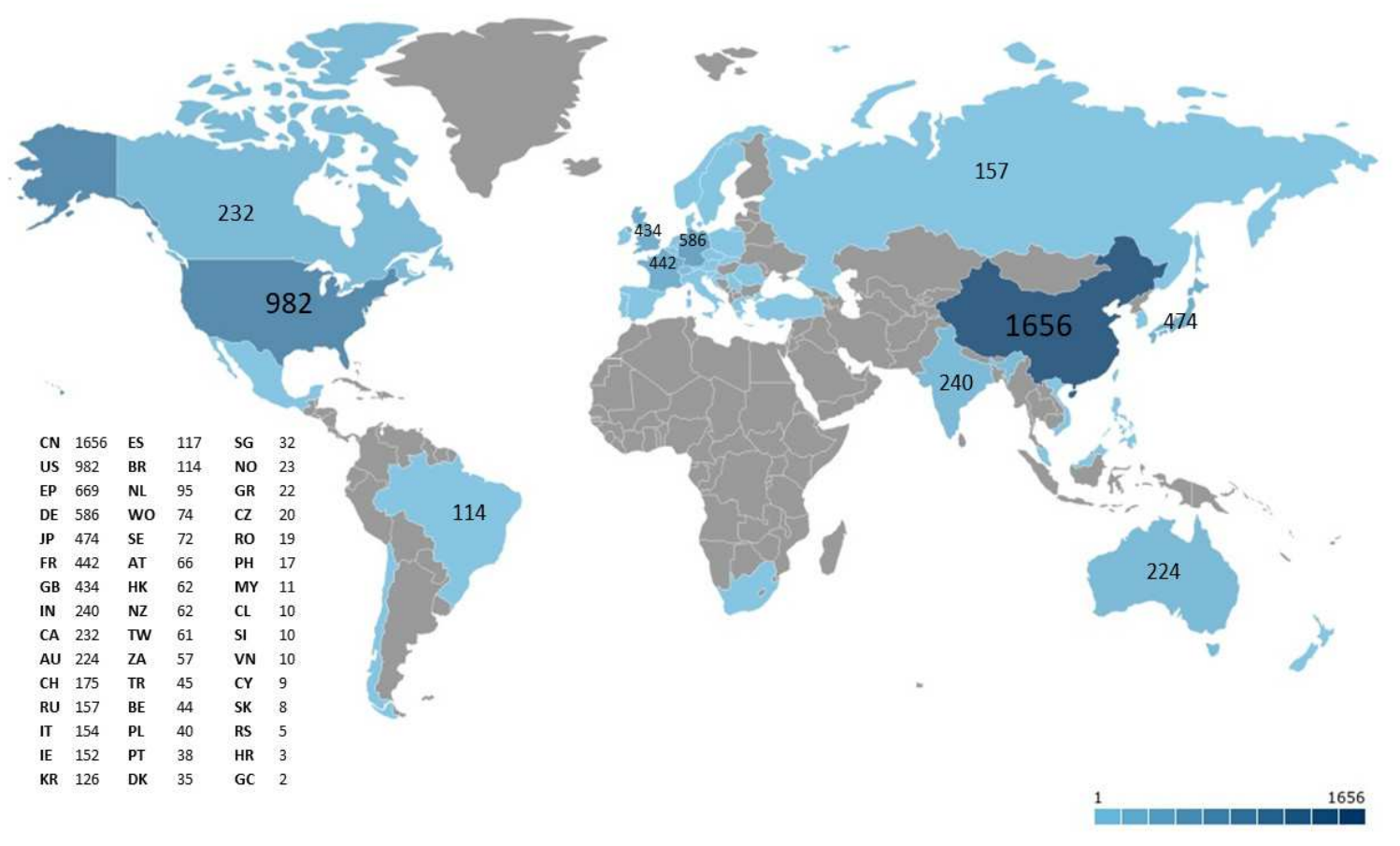

Fonte: Elaborada pelos autores deste artigo (2021)

Quando são comparadas as Famílias de patentes por primeiro país de prioridade com as Famílias de patentes ativas (pedidos e concedidas) por país de proteção, verifica-se que a China $(\mathrm{CN})$ se destaca nas duas situações, pois, além de ser o país no qual mais se desenvolvem tecnologias relacionadas a ventiladores/respiradores mecânico, também é o maior mercado de exploração da tecnologia, uma vez que detém o maior número de família de patentes com direitos de propriedade industrial vigentes, correspondendo a $20,7 \%$ das famílias de patentes ativas. Em seguida, se destacam os Estados Unidos (US), com 12,3\%, a União Europeia, representada pela Organização Europeia de Patentes (EP), com 8,4\%, e a Alemanha (DE), com 7,3\%. 
Continuando com a comparação entre os números apresentados nas Figuras 4 e 5, destacam-se os dados referentes ao Canadá (CA) e à Índia (IN), pois, apesar de os dois países apresentarem números baixos de prioridades de famílias de patentes, 21 e 18, respectivamente, eles se destacam entre os 10 maiores mercados de exploração da tecnologia aqui apresentada, sendo a IN em $8^{\circ}$, com 240 (3\%) famílias de patentes, e o CA, com 232 (2,9\%).

\section{Considerações Finais}

A pandemia do Coronavírus, iniciada em 2020, provocou uma crise mundial no sistema de saúde, ocasionando milhões de mortes e filas de pacientes aguardando uma vaga de UTI para serem atendidos, especialmente com Síndromes Respiratórias Agudas. Tornou-se evidente a necessidade de se obter recursos materiais e humanos habilitados para lidar com as situações adversas do quadro clínico dos pacientes acometidos pela Covid-19. Especialmente nos países em desenvolvimento, já que seus sistemas de saúde não dispunham de tecnologias e de leitos de UTI suficientes para atender à demanda da população, a situação pandêmica e o número de mortes se tornavam mais críticos.

Alinhado a esse contexto, inúmeras iniciativas de pesquisadores e grupos de trabalho começaram já no início da pandemia a produzir ou desenvolver soluções que ajudassem a desafogar os sistemas de saúde de seus países, especialmente para o tratamento da Covid-19. Assim também foi no Brasil, quando se iniciou o desenvolvimento de equipamentos de proteção individual e de dispositivos de ventilação mecânica, pois eles passaram a ser copiados, adaptados, recriados e/ou criados.

Nessa perspectiva, o presente estudo objetivou apresentar, por meio de informações contidas em base de dados de patentes, uma visão prospectiva de tecnologias relacionadas a ventiladores ou respiradores artificiais mecânicos, com vistas a dar subsídios à tomada de decisões de pesquisadores autoridades em saúde. A busca do estado da arte, especialmente o mapeamento tecnológico ou a prospecção tecnológica, permite analisar, sob diferentes perspectivas, o cenário do desenvolvimento de tecnologias e de proteção destas pelos direitos de propriedade industrial, permitindo, assim, tomar decisões sobres as possiblidades de licenciamento, transferência de tecnologia, apropriação ou criação de novas tecnologias relacionadas ao tema pesquisado.

Os resultados da pesquisa sobre patentes de respiradores artificiais e ventiladores mecânicos possibilitaram apresentar o mapeamento da produção de patentes relacionadas à tecnologia em estudo, apontando a evolução do desenvolvimento da tecnologia no decorrer do tempo, os principais players envolvidos no desenvolvido de tal tecnologia, os inventores associados a estas, assim como os principais países onde as tecnologias foram desenvolvidas e protegidas.

Observou-se que documentos de patentes de respiradores e ventiladores artificiais mecânicos já podiam ser encontrados desde 1909, mas é nos últimos anos que o número famílias de patentes dessa tecnologia tem crescido, sendo seu pico atingido em 2019, com 500 documentos. No entanto, em todo período pesquisado, existem apenas 14 famílias de patentes requeridas por residentes brasileiros no INPI, apesar de existirem 114 famílias de patentes ativas e, portanto, que detêm direitos de propriedade industrial dos seus titulares resguardados. Há, porém, mais de 5.900 famílias de patente que podem ser exploradas no território brasileiro sem infringir 
quaisquer direitos, seja porque estão inativas por quaisquer motivos ou porque não foram requeridas proteção no Brasil.

Por fim, não há como negar que as iniciativas de pesquisa e desenvolvimento de respiradores e ventiladores artificiais mecânicos têm sido pouco exploradas, quando comparadas a necessidade de utilização dessa tecnologia na composição da estruturação do sistema de saúde do país. Nesse contexto, estudos como este e ainda mais aprofundados nas especificações técnicas e funcionais dessa tecnologia devem ser recomendados aos pesquisadores, a fim de auxiliarem no direcionamento de pesquisas focadas no desenvolvimento de produtos novos e dotados de atividade inventiva, para que possam ser patenteados e trilhem o caminho da inovação, não somente para a demanda tecnológica do mercado nacional, mas também para fomentar o desenvolvimento social, econômico, científico e tecnológico do país.

\section{Perspectivas Futuras}

A urgente demanda mundial por novos ventiladores ou respiradores pulmonares para atender às necessidades dos sistemas de saúde e para o enfrentamento crítico da pandemia fez surgir uma série de iniciativas ao redor do mundo de pesquisas que promovam o desenvolvimento de novas tecnologias médicas. Mas, como o ritmo normal de produção desse tipo de equipamento, segundo Santos (2020), é insuficiente para atender a tempo a quantidade estimada de casos graves de Covid-19 que chegarão nas UTIs, as iniciativas para facilitar a apropriação $e$ a utilização de ventiladores e respiradores já aprovados em determinados países também têm sido adotadas no Brasil.

Desse modo, algumas tendências já têm sido concretizadas, conforme foram apresentadas neste trabalho, como a flexibilização nos processos de registro na Anvisa e algumas iniciativas de desenvolvimento de novos equipamentos do tipo ventiladores e respiradores pulmonares. Portanto, a curto prazo, espera-se que a produção de ventiladores e de respiradores pulmonares seja intensificada no território brasileiro, de modo a dar maior subsídios aos hospitais no que tange à disponibilização desses equipamentos em suas UTIs. A longo prazo, estima-se que a capacidade de aprendizagem tecnológica de pesquisadores atuantes no campo das tecnologias médicas seja ampliada e permita gerar novas tecnologias e a disseminação desse conhecimento específico para outras gerações de pesquisadores.

De modo prático, as informações apresentadas aqui podem servir de suporte para o desenvolvimento de tecnologias que atendam às demandas por esses equipamentos tecnológicos nos hospitais brasileiros, por exemplo, a inciativa de um grupo de pesquisadores no Estado da Bahia que tem trabalhado para desenvolver um respirador artificial de baixo custo, adaptado com Reanimador Manual (Ambu), para uso em pacientes com insuficiência respiratória vítimas da Covid-19.

Outro ponto importante destacado pelo grupo de trabalho é a integração de conhecimento entre as áreas na busca de um denominador comum para aplicação. Assim, o uso da tecnologia e a abrangência dos conhecimentos poderão ser úteis não somente para o desenvolvimento de respiradores e sim para outros equipamentos, tornando-os acessíveis às comunidades mais carentes. 
Futuros trabalhos podem ser desdobrados a partir deste para dar continuidade a outras vertentes desta pesquisa, pois, apesar das pesquisas existentes e em andamento, uma série de graves problemas associados à pandemia de Covid-19 carece de tecnologias capazes de minimizar seus impactos.

\section{Referências}

ANVISA - AGÊNCIA NACIONAL DE VIGILÂNCIA SANITÁRIA. Ventiladores pulmonares: Brasil amplia a produção. 2020. Disponível em: encurtador.com.br/evGR2. Acesso em: 16 maio 2021.

ARUNKUMAR, K. E. et al. Forecasting the dynamics of cumulative COVID-19 cases (confirmed, recovered and deaths) for top-16 countries using statistical machine learning models: Auto-Regressive Integrated Moving Average (ARIMA) and Seasonal Auto-Regressive Integrated Moving Average (SARIMA). Applied Soft Computing, [s.l.], v. 103, p. 107161, 2021. Disponível em: https:/www. sciencedirect.com/science/article/pii/S1568494621000843. Acesso em: 16 maio 2021.

CARVALHO, C. R. R.; TOUFEN JÚNIOR, C.; FRANCA, S. A. Ventilação mecânica: princípios, análise gráfica e modalidades ventilatórias. J. Bras. Pneumol., São Paulo, v. 33, supl. 2, p. 54-70, jul. 2007. Disponível em: https://doi.org/10.1590/S1806-37132007000800002. Acesso em: 12 set. 2020.

CLARIVATE. Web of Science. [2021]. Disponivel em: https://apps-webofknowledge. ez278.periodicos.capes.gov.br/WOS_GeneralSearch_input.do?product=WOS\&search_ mode $=$ GeneralSearch. Acesso em: 16 maio 2021 .

FIOCRUZ - FUNDAÇÃO OSVALDO CRUZ. Observatório COVID-19: informação para ação. [2021]. Disponível em: https://portal.fiocruz.br/sites/portal.fiocruz.br/files/documentos/serie_historica_ leitos_uti_covid-19_adultos.pdf. Acesso em: 15 mar. 2021.

GALVÃO, Antonio Carlos Filgueira et al. O quadro recente de emprego dos mestres e doutores titulados no Brasil. Parcerias Estratégicas, [s.l.], v. 21, n. 43, p. 147-172, 2016. Disponível em: http://seer.cgee.org.br/index.php/parcerias_estrategicas/article/viewFile/839/768. Acesso em: 12 mar. 2021.

INPI - INSTITUTO NACIONAL DA PROPRIEDADE INDUSTRIAL. (2020). Disponível em: http:// https://www.gov.br/inpi. Acesso em: 10 mar. 2021.

ISER, B. P. M. et al. Definição de caso suspeito da COVID-19: uma revisão narrativa dos sinais e sintomas mais frequentes entre os casos confirmados. Epidemiol. Serv. Saúde, Brasília, v. 29, n. 3, p. 202-233, 2020. Disponível em: https://www.scielosp.org/pdf/ress/2020.v29n3/e2020233/pt. Acesso em: 9 mar. 2021.

KACMAREK, R. M. The mechanical ventilator: past, present and future. Respiratory Care, [s.l.], v. 56, n. 8, p. 1.170-1.180, ago. 2011. Disponível em: http://rc.rcjournal.com/content/56/8/1170.full. Acesso em: 16 maio 2021.

PAHO - PLISA HEALTH INFORMATION PLATFORM FOR THE AMERICAS. [World Health Organization - WHO]. Folha informativa - COVID-19 (doença causada pelo novo coronavírus). 2020. Disponível em: https:/www.paho.org/bra/index.php?option=com_content\&vie $w=$ article\&id= 6101: covid19\&Itemid=875. Acesso em: 12 set. 2020. 
PIRES, E. A.; RIBEIRO, N. M.; QUINTELLA, C. M. Sistemas de Busca de Patentes: análise comparativa entre Espacenet, Patentscope, Google Patents, Lens, Derwent Innovation Index e Orbit Intelligence. Cadernos de Prospecção, Salvador, v. 13, n. 1, p. 13-29, março, 2020. Disponível em: https://portalseer.ufba.br/index.php/nit/article/view/35147. Acesso em: 12 set. 2020.

PLETSCH-ASSUNÇÃO, R.; CUNHA, M. R.; VIEIRA, S. R. R. Efetividade de um protocolo de ventilação mecânica não-invasiva em pacientes internados em uma unidade de terapia intensiva de um hospital geral. Revista Multidisciplinar da Saúde, [s.l.], v. 1, n. 1, p. 58-65, 2019. Disponível em: https://revistas.anchieta.br/index.php/RevistaMultiSaude/article/view/1467. Acesso em: 2 set. 2020.

RACHE, Beatriz et al. Necessidades de Infraestrutura do SUS em Preparo ao COVID19: Leitos de UTI, Respiradores e Ocupação Hospitalar. Instituto de Estudos para Políticas de Saúde. IEPS Instituto de Estudos para Políticas de Saúde, [s.l.], Nota Técnica n. 3, 2020. Disponível em: http://www.epsjv.fiocruz.br/sites/default/files/files/NT3\%20vFinal.pdf. Acesso em: 2 set. 2020.

\section{RUBENFELD, Gordon D. Epidemiology of acute lung injury. Read Online: Critical Care}

Medicine| Society of Critical Care Medicine, [s.l.], v. 31, n. 4, p. S276-S284, 2003. Disponível em: https://journals.lww.com/ccmjournal/Abstract/2003/04001/Epidemiology_of_acute_lung_ injury.17.aspx. Acesso em: 9 mar. 2021.

SANTOS, Cristina d'Urso S. M. Observatório de Tecnologias Associadas à Covid-19: pedidos de patente de ventiladores pulmonares. [S.l.]: Ministério da Economia; Instituto Nacional da Propriedade Industrial, 2020. Disponível em: https://www.gov.br/inpi/pt-br/servicos/patentes/ tecnologias-para-covid-19/Arquivos\%20Textos/Estudo_2_ventiladores_pulmonares.pdf. Acesso em: 17 maio 2021.

SILVA, M. B. et al. Enfrentamento à Covid-19: um mapeamento das patentes de ventiladores mecânicos. Cadernos de Prospecção, Salvador, v. 13, p. 526-542, 2020. Disponível em: https:// periodicos.ufba.br/index.php/nit/article/view/36186. Acesso em: 16 maio 2021.

SPRICIDO, Tiago Vinícius do N. Proposta de uso de motor radial para desenvolvimento de respiradores mecânicos de baixo custo com bolsas de AMBU. 2021. 30p. Trabalho de Conclusão de Curso (Graduação em Engenharia Mecânica) - Centro Universitário de Maringá, Maringá, 2021. Disponível em: http://rdu.unicesumar.edu.br/handle/123456789/7698. Acesso em: 31 maio 2021.

SISTEMA UNIVERSIDADE ABERTA DO SUS - UNA-SUS. Organização Mundial de Saúde declara pandemia do novo Coronavírus. UNA-SUS, [s.l.], 11 de março de 2020. Disponível em: https:// www.unasus.gov.br/noticia/organizacao-mundial-de-saude-declara-pandemia-de-coronavirus. Acesso em: 9 mar. 2021.

SUZUKI, H.; QUINTELLA, C. M. Reflexões sobre o grau de (des)preparo tecnológico do país evidenciadas pela COVID-19. E o que nós (autores e leitores da revista Cadernos de Prospecção) temos a ver com isso? Cadernos de Prospecção, Salvador, v. 13, p. 311-312, 2020. Disponível em: https://periodicos.ufba.br/index.php/nit/article/view/36326. Acesso em: 16 maio 2021.

TOUFEN JÚNIOR, C.; CARVALHO, C. R. R. Ventiladores mecânicos. J. Bras. Pneumol., São Paulo, v. 33, supl. 2, p. 71-91, July 2007. Disponível em: http://www.scielo.br/scielo.php?script=sci_arttext\& pid=S1806-37132007000800003 \&lng=en\&nrm=iso. Acesso em: 2 set. 2020.

WHO - WORLD HEALTH ORGANIZATION. Influenza pandemic preparedness plan: the role of WHO and guidelines for national and regional planning. World Health Organization, Geneva, Switzerland, April, 1999. Disponível em: https://apps.who.int/iris/handle/10665/66155. Acesso em: 3 set. 2020. 
WHO - WORLD HEALTH ORGANIZATION. What is a pandemic? [2020a]. Disponivel em: https:// www.who.int/csr/disease/swineflu/frequently_asked_questions/pandemic/en/. Acesso em: 12 set. 2020.

WHO - WORLD HEALTH ORGANIZATION. WHO Report on Global Surveillance of Epidemicprone Infectious Diseases - Influenza. [2020b]. Disponível em: https:/www.who.int/csr/resources/ publications/influenza/CSR_ISR_2000_1/en/. Acesso em: 12 set. 2020.

WHO - WORLD HEALTH ORGANIZATION. WHO Coronavirus (COVID-19) Dashboard. [2021]. Disponível em: https://covid19.who.int/table/. Acesso em: 16 maio 2021.

WIPO - WORLD INTELLECTUAL PROPERTY ORGANIZATION. Guide to the International

Patent Classification. 2020. Disponível em: https://www.wipo.int/edocs/pubdocs/en/wipo_guide_ ipc_2020.pdf. Acesso em: 3 set. 2020.

XU, Zhe et al. Pathological findings of COVID-19 associated with acute respiratory distress syndrome. The Lancet Respiratory Medicine, [s.l.], v. 8, n. 4, p. 420-422, 2020. Disponível em: https://www. thelancet.com/journals/lanres/article/PIIS2213-2600(20)30076-X/fulltext. Acesso em: 2 nov. 2020.

ZHANG, L.; LI, L.; LI, T. Patent Mining: A Survey. ACM SIGKDD Explorations Newsletter, [s.l.], v. 16, n. 2, p. 1-19, 2015. Disponível em: https://dl.acm.org/doi/10.1145/2783702.2783704. Acesso em: 24 set. 2020 .

\section{Sobre os Autores}

\section{Edilson Araújo Pires}

E-mail: edilson@ufrb.edu.br

ORCID: https://orcid.org/0000-0002-8258-1739

Doutor em Ciência da Propriedade Intelectual.

Endereço profissional: Rua Rui Barbosa, n. 710, Centro, Cruz das Almas, BA. CEP: 44380-000.

\section{Jamile de Jesus Souza Andrade}

E-mail: jamilesouza.unica@gmail.com

ORCID: https://orcid.org/0000-0002-6124-0492

Especialista em Gestão de Micro e Pequenas Empresas.

Endereço profissional: Avenida Dois de Julho, n. 31, Centro, Santo Antônio de Jesus, BA. CEP: 44430-074.

\section{Fábio André Lora}

E-mail: fabio.lora@ufrb.edu.br

ORCID: https://orcid.org/0000-0001-9951-1916

Doutor em Engenharia de Minas, Metalúrgica e Materiais.

Endereço profissional: Rua Godofredo Rebello de Figueiredo Filho, n. 697, Bairro SIM, Feira de Santana, BA. CEP: 44085-132. 